Published in final edited form as:

JACC Cardiovasc Interv. 2019 February 25; 12(4): 362-369. doi:10.1016/j.jcin.2018.11.008.

\title{
Impact of Short-Term Complications on Mortality and Quality of Life after TAVR
}

\author{
Suzanne V. Arnold, MD, MHA ${ }^{1}$, Yiran Zhang, MS ${ }^{2}$, Suzanne J. Baron, MD, MSc ${ }^{1}$, Thomas C. \\ McAndrew, PhD $^{2}$, Maria C. Alu, MS $^{2}$, Susheel K. Kodali, MD², Samir Kapadia, MD ${ }^{3}$, Vinod H. \\ Thourani, MD $^{4}$, D. Craig Miller, MD $^{5}$, Michael J. Mack, MD ${ }^{6}$, Martin B. Leon, MD ${ }^{2}$, and David \\ J. Cohen, MD, MSc ${ }^{1}$
}

${ }^{1}$ Saint Luke's Mid America Heart Institute and University of Missouri-Kansas City, Kansas City, MO

${ }^{2}$ Columbia-Presbyterian Hospital, New York, NY

${ }^{3}$ Cleveland Clinic Foundation, Cleveland, $\mathrm{OH}$

${ }^{4}$ Medstar Washington Hospital Center/Georgetown University, Washington, DC

${ }^{5}$ Stanford University Medical School, Stanford, CA

${ }^{6}$ Baylor Scott and White Health, Plano, TX

\section{Abstract}

Background: Prior studies have examined the mortality and cost implications of various complications of TAVR. However, many of these complications may primarily impact patients' quality of life after TAVR, which has not been previously studied.

Objective: To examine the independent association of short-term complications of TAVR with survival and quality of life at 1 year.

Methods: Among patients at intermediate or high surgical risk who underwent TAVR as part of the PARTNER 2 studies and survived 30 days, we examined the association between complications within the 30 days after TAVR and mortality and quality of life at 1 year. Quality of life was assessed with the Kansas City Cardiomyopathy Questionnaire (KCCQ) and the Short

\footnotetext{
Address for Correspondence: Suzanne V. Arnold MD, MHA, Saint Luke's Mid America Heart Institute, 4401 Wornall Rd, Kansas City, MO 64111, Phone: 816-932-8606, Fax: 816-932-5613, suz.v.arnold@ gmail.com.

Publisher's Disclaimer: This is a PDF file of an unedited manuscript that has been accepted for publication. As a service to our customers we are providing this early version of the manuscript. The manuscript will undergo copyediting, typesetting, and review of the resulting proof before it is published in its final citable form. Please note that during the production process errors may be discovered which could affect the content, and all legal disclaimers that apply to the journal pertain.

Disclosures:

Dr. Baron has received consulting income from Edwards Lifesciences and St. Jude Inc.

Dr. Kodali has received consulting income from Edwards Lifesciences, Medtronic; and is an advisory board member for Thubrikar Aortic Valve, Inc., Paieon Medical, and St. Jude Medical.

Dr Thourani served as advisor for Edwards Lifesciences, Abbott Vascular, Boston Scientific, and Gore Vascular.

Drs. Mack and Leon received travel reimbursements from Edwards Lifesciences for activities related to their positions on the Executive Committee of the PARTNER Trial.

Dr. Cohen has received research grant support from Edwards Lifesciences, Medtronic, Boston Scientific, and Abbott Vascular and consulting fees from Medtronic and Edwards Lifesciences. The other authors report no conflicts.
} 
Form-12. Complications assessed included major and minor stroke, life-threatening and major bleeding, vascular injury, stage 3 acute kidney injury [AKI], new pacemaker, and mild and moderate/severe paravalvular leak (PVL). We used multivariable models that included all complications as well as baseline clinical factors to examine the independent association of each complication with outcomes.

Results: Among 3763 TAVR patients, we found that major stroke and stage 3 AKI were associated with markedly increased risk of 1-year mortality (adjusted HRs 5.4 [95\% CI 3.1-9.5] and 4.9 [95\% CI 2.7-8.8], respectively) as well as poorer quality of life among survivors (reductions in 1-year KCCQ overall summary score of 15.1 [95\% CI -24.8 to -5.3] and 14.7 [95\% CI -25.6 to -3.8] points, respectively). Moderate or severe PVL, life-threatening bleeding, and major bleeding were each associated with a more modest increase in mortality and decrement in quality of life whereas mild PVL was associated with a small decrease in quality of life. After adjusting for baseline characteristics and other complications, need for new pacemaker, minor stroke, and vascular injury were not independently associated with poor outcomes.

Conclusions: Among patients undergoing TAVR, similar events are associated with increased mortality and impaired quality of life at 1-year. These results suggest that despite considerable progress, efforts to further reduce stroke, AKI, bleeding, and moderate/severe PVL are likely to yield important clinical benefits and remain key targets for device iteration and procedural improvement.

\section{CONDENSED ABSTRACT}

Among 3763 TAVR patients, major stroke and kidney injury were both independently associated with a markedly increased risk of 1-year mortality and a large reduction in quality of life (among survivors). Bleeding and paravalvular leak were associated with a smaller increase in the risk of death and reduction in late quality of life. Pacemakers, minor stroke, and vascular complications, on the other hand, had no significant impact on long-term outcomes (in the absence of other complications). These data provide insights into the complications that have the greatest impact on patients' recovery after TAVR and should help focus future efforts to improve care.

\section{Keywords}

transcatheter aortic valve replacement; complications; quality of life

\section{INTRODUCTION}

Transcatheter aortic valve replacement (TAVR) has been shown to effectively improve both survival and quality of life compared with medical therapy in patients with aortic stenosis unsuitable for surgical valve replacement $(1,2)$ and to have outcomes that are similar or superior to those of surgical valve replacement in high- $(3,4)$ and intermediate-risk patients $(5,6)$. However, these improvements in survival and quality of life represent average effects over a population, and a substantial minority do not benefit significantly from the procedure (7-9). While patient factors such as advanced age, comorbidities, and frailty contribute substantially to poor long-term outcomes after TAVR $(7,9)$, complications likely also impair recovery. Prior studies of complications of TAVR have focused on their associations with mortality (10-15); however, many of these complications may primarily impact patients' 
quality of life after TAVR. Furthermore, many of these complications also coexist, and their independent contributions to long-term outcomes have not been fully explored. Therefore, we sought to use data from the Placement of AoRTic TraNscathetER Valve (PARTNER) 2 studies, including the non-randomized registries, to estimate the impact of short-term complications on 1-year mortality and quality of life after TAVR. By doing so, we hoped to provide important insight as to which complications have the greatest impact on key outcomes in order to help guide future efforts for procedural improvement.

\section{METHODS}

\section{Study Design and Population.}

Our analytic cohort was derived from the PARTNER 2 studies, which included patients undergoing TAVR as part of the PARTNER 2A randomized trial and number of nonrandomized registries (ClinicalTrials.gov Identifier: NCT01314313). Included patients had severe aortic stenosis (mean gradient $>40 \mathrm{mmHg}$ or jet velocity greater than $4.0 \mathrm{~m} / \mathrm{s}$ and aortic valve area $\$ 0.8 \mathrm{~cm}^{2}$ or $<0.5 \mathrm{~cm}^{2} / \mathrm{m}^{2}$ ) in addition to New York Heart Association Functional Class II or greater symptoms. The PARTNER 2A clinical trial and SAPIEN 3 intermediate-risk registry $(6,16)$ enrolled patients who were felt to be at intermediate surgical risk (predicted risk of 30-day mortality 4\%-8\%, based on either the Society of Thoracic Surgeons [STS] mortality risk score or clinical assessment by a multidisciplinary heart team). There were a number of nested registries within PARTNER 2 and SAPIEN 3 that also enrolled high risk (STS $>8 \%$ ) and inoperable patients (heart team estimation of $250 \%$ risk of death or serious, irreversible morbidity with surgery). While the studies generally followed similar protocols with respect to inclusion and exclusion criteria, baseline data collection, and follow-up, the PARTNER 2A and 2B studies used the SAPIEN-XT valve while the SAPIEN 3 studies used the SAPIEN 3 valve. Key exclusion criteria included baseline creatinine $>3 \mathrm{mg} / \mathrm{dL}$ or renal replacement therapy, mixed aortic valve disease with predominant aortic regurgitation, congenital unicuspid or bicuspid aortic valve, and left ventricular ejection fraction $<20 \%$. All studies were approved by the institutional review board at each site, and written informed consent was obtained from all patients.

\section{Short-term Complications.}

Complications within the 30-days after TAVR were adjudicated by a clinical events committee according to the Valve Academic Research Consortium-2 definitions (17) and included the following: death, major stroke, minor stroke, life-threatening bleeding, major bleeding (distinct from life-threatening bleeding), major vascular complication, new permanent pacemaker, and stage 3 acute kidney injury (AKI) (Supplemental Table 1). The severity of paravalvular leak (PVL) on the 30-day follow-up echocardiogram was assessed as none, trace, mild, moderate, or severe by an independent core laboratory.

\section{Outcomes.}

As the assessment of PVL required an echocardiogram at 30 days after TAVR, only patients who survived at least 30 days after TAVR were included in the analysis. Mortality was assessed from 30 days to 1 year after TAVR. As a sensitivity analysis, to examine the association of complications with early or late mortality, we examined time to death for all 
patients from the time of TAVR. Of note, PVL was excluded from this analysis since it was first assessed by the core laboratory at the 30-day echocardiogram. Disease-specific quality of life was assessed at baseline and 1 year using the Kansas City Cardiomyopathy Questionnaire (KCCQ), a disease-specific health status measure that has been validated in patients with aortic stenosis.(18) The KCCQ includes 5 key domains of health status (physical function, social function, symptoms, self-efficacy, and quality of life) that are combined into a single overall summary score (KCCQ-OS), which served as the primary outcome of our study. Scores on the KCCQ-OS scale range from 0 to 100, with higher scores indicating fewer symptoms and functional limitations. KCCQ-OS scores roughly correlate with New York Heart Association Functional Class as follows: Class I $\approx$ KCCQOS 75-100; Class II $\approx 60-74$; Class III $\approx 45-59$; and Class IV $\approx 0-44$ (19), and changes of 5,10 , and 20 points correspond with small, moderate, and large clinical improvements, respectively.(20) Generic quality of life was assessed with the Medical Outcomes Study Short-Form 12 (SF-12) questionnaire(21) physical (PCS) and mental (MCS) component summary scores, which are scaled to overall US norms of 50 with standard deviations of 10 . Higher scores indicate better quality of life, and the minimum clinically important differences for the PCS and MCS are $~ 2.5$ points.

\section{Statistical Analysis.}

In unadjusted analyses, we first constructed Kaplan Meier mortality curves for patients with versus without each short-term complication and compared groups using the log-rank statistic. Of note, for bleeding, stroke, and PVL, these were 3-level comparisons (e.g., none vs. mild vs. moderate/severe PVL). Among patients who survived to 1 year, we then examined the mean change in KCCQ-OS, SF-12 PCS, and SF-12 MCS from baseline to 1 year among patients with no short-term complications and those with each of the 8 complications. As these were not mutually exclusive groups, no statistical comparisons were made.

We used multivariable models in order to examine the independent association between each of the various short-term complications and both mortality and quality of life. For the mortality analysis, we used a Cox proportional hazards model, whereas for quality of life, we used linear regression models. Models included all complications along with patient factors that have previously been associated with risk of TAVR complications (22): age, sex, prior coronary artery bypass graft surgery, peripheral vascular disease, diabetes, STS mortality risk score, nontransfemoral access, and baseline KCCQ score (or SF-12 PCS or MCS for their respective models). As complications do not always occur in isolation (e.g., one patient may have both a major bleed and AKI), all complications were included simultaneously in the models, which allowed us to examine the survival and quality of life decrement associated with each complication, independent of other complications that may also have occurred in the same patient. All analyses were performed with SAS version 9.4 (SAS institute, Cary, North Carolina). 


\section{RESULTS}

\section{Study Cohort and 30-day Complications.}

Among 3897 patients with severe symptomatic aortic stenosis who were enrolled in one of the PARTNER 2 studies and underwent TAVR, 3763 survived to 30 days and formed our primary analytic cohort. The mean age of the cohort was 82 years, $42 \%$ were women, mean STS mortality risk score was $7.4 \%$, and $80 \%$ of patients had transfemoral access for their TAVR. Mean KCCQ-OS prior to TAVR was 49.6 points, mean SF-12 PCS was 35.1 points (1.5 SD below US population mean), and mean SF-12 MCS was 48.5 points. The frequency of short-term complications is shown in Table 2. Mild PVL was the most common complication at $34.7 \%$. Bleeding was also fairly common, with $16.8 \%$ of patients having a major bleed and $6.9 \%$ having a life-threatening bleed. Major strokes, minor strokes, and stage 3 AKI were rare, occurring in $1.5 \%, 1.4 \%$, and $1.0 \%$ of patient, respectively.

\section{Mortality.}

Between 30 days and 1 year, 360 patients died (9.6\% mortality rate from 30 days to 1 year), which occurred more commonly in patients who had a short-term complication than those who did not $(12.1 \%$ vs. $6.8 \%, \mathrm{p}<0.0001)$. The unadjusted Kaplan-Meier survival curves for patients with versus without each of the complications are shown in Supplemental Figure 1. In terms of individual complications, patients with major strokes and stage 3 AKI had the highest unadjusted 1-year mortality rates at $49.3 \%$ and $51.7 \%$, respectively (Table 3 ). In unadjusted analyses, major and life-threatening bleeds, new pacemaker, and moderate/severe PVL were also associated with increased mortality.

In the multivariable model that accounted for concomitant complications and patient factors, major stroke and stage 3 AKI continued to be associated with a markedly increased risk of mortality (major stroke: adjusted hazard ratio [HR] 5.4, 95\% CI 3.1-9.5; AKI: adjusted HR 4.9, 95\% CI 2.7-8.8; Table 4). Life-threatening bleeding, major bleeding, and moderate/ severe PVL were also independently associated with increased mortality, but to a lesser extent (life-threatening bleed: adjusted HR 1.6, 95\% CI 1.0-2.5; major bleed: adjusted HR 1.5, 95\% CI 1.1-2.0; moderate/severe PVL: adjusted HR 1.9, 95\% CI 1.3-2.7). On the other hand, after adjusting for other complications and patient factors, minor strokes, major vascular complications, new pacemakers, and mild PVL were not independently associated with increased risk of 1-year death. In a sensitivity analysis examining the association of complications with mortality (including in-hospital deaths), results were similar, although the association of life-threatening bleeding with mortality was stronger (HR 3.2, 95\% CI 2.5-4.3; Supplemental Table 2)

\section{Quality of Life.}

Among 3403 patients eligible for the 1-year quality of life analysis, 2875 and 2147 completed the 1-year KCCQ (84.5\%) and SF-12 (63.1\%), respectively. Patients who survived but were missing KCCQ data were similar to those in the analytic cohort but were more likely to be older with higher STS risk scores (Supplemental Table 3); moreover, completion rates for the KCCQ and SF-12 were slightly higher among patients with versus without a complication (KCCQ: $85.9 \%$ vs. $82.6 \%$, p=0.008; SF-12: $65.4 \%$ vs. $60.1 \%$, 
$\mathrm{p}=0.002$ ). The unadjusted mean changes in KCCQ-OS, SF-12 PCS, and SF-12 MCS from baseline to 1 year among surviving patients are shown in Table 3. Surviving patients who had no short-term complications had an average increase in KCCQ-OS of 26.6 points with improvements of 5.0 points and 3.8 points for the SF-12 PCS and SF-12 MCS, respectively. Patients who had a major stroke or stage 3 AKI and survived to 1 year had, on average, small improvements in KCCQ-OS of 5.4 and 2.7 points, respectively, and worse SF-12 PCS and MCS scores (PCS: -1.7 and -3.6 ; MCS: -0.7 and 1.5, respectively). Patients with the other complications had mean improvements that were slightly less than those without complications (KCCQ-OS: 20-23 points, PCS: 2-4 points, MCS: 1-3 points).

In the multivariable analysis that accounted for concomitant complications and patient factors, major stroke and stage $3 \mathrm{AKI}$ were associated with a marked attenuation of the extent of improvement in the KCCQ-OS at 1 year (major stroke: -15.1 points, 95\% CI -24.8 to -5.3 ; AKI: -14.7 points, $95 \% \mathrm{CI}-25.6$ to -3.8 ; Table 4 ). Life-threatening bleeding and mild PVL were associated with small but statistically significant reductions in recovery of the KCCQ-OS at 1 year, whereas minor strokes, major bleeding, major vascular complications, moderate/severe PVL, and new pacemakers were not associated with significant reductions in the KCCQ-OS at 1 year after TAVR. Similar trends were seen in the SF-12 PCS and MCS, although only major stroke (PCS: $-5.1,95 \%$ CI -10.18 to -0.03 ), life-threatening bleeding (PCS $-2.5,95 \% \mathrm{CI}-4.4$ to - 0.7), major bleeding (PCS: $-1.2,95 \%$ CI -2.3 to 0.0 ), AKI (PCS $-6.4,95 \% \mathrm{CI}-12.3$ to -0.5 ) and moderate/severe PVL (MCS: $-2.1,95 \%$ CI -3.9 to -0.3 ) reached statistical significance (Supplemental Table 3).

\section{DISCUSSION}

In a large cohort of patients who underwent TAVR and survived 30 days, we found that major stroke and stage $3 \mathrm{AKI}$, while uncommon, were associated with a markedly increased risk of 1-year mortality as well as a large reduction in quality of life (among surviving patients). Bleeding (both life-threatening and major) and moderate/severe PVL all occurred more commonly and were associated with a small but significantly increased risk of death and a small reduction in late quality of life. On the other hand, complications such as minor strokes, new pacemakers, and vascular complications (in the absence of life threatening or major bleeding) had no significant impact on long-term mortality or quality of life recovery. These data provide important insights into the complications that have the greatest impact on patients' recovery after TAVR and could be used to help guide future device iterations and peri-procedural management.

\section{Clinical Implications.}

In the early TAVR experience, complications such as vascular injury, stroke, paravalvular regurgitation, and the need for pacemaker implantation were common $(1,23)$. Over the past decade, however, device modifications (e.g. larger range in valve sizes, reduced valve profile, external sealing skirts) along with use of routine CT sizing and 3-D imaging for procedural planning and increased operator experience have led to substantial reductions in most complications (24). Nonetheless, serious complications have not been eliminated and certain complications such as pacemaker and major bleeding continue to occur in $>10 \%$ of 
patients (24). Pacemakers have been a key area of interest given their relative frequency and the fact that rates appear to differ across TAVR devices $(14,25)$. While reducing the need for pacemakers after TAVR should reduce length of stay and costs (22), our analysis shows that this complication has little effect on either survival or quality of life. In contrast, our findings suggest that bleeding events remain an area of concern, since they are both common and prognostically important, and often occur in the absence of vascular complications. Importantly, bleeding occurred both with and without vascular complications, with only $32 \%$ of bleeding complications occurring during the TAVR procedure (61\% post-procedure but before discharge and 7\% after discharge). With increasing concern about valve thrombosis $(26,27)$, there may be increasing use of oral anticoagulants after TAVR, thus exposing patients to further risk of post-procedural bleeding. Our results suggest that we must use caution with this approach, as decreasing the risk of these ischemic events may be counterbalanced by an increased risk of bleeding, both of which have potential to impact patients' recovery.

\section{Prior Studies.}

Our results support and extend prior studies of the association between short-term complications of TAVR and outcomes, which have demonstrated increased mortality in patients who experience major bleeding, major strokes, renal failure, and moderate/severe PVL (10-15). Although at least one prior study has shown increased risk of mortality after major vascular complications, this study failed to evaluate other complications as potential confounders of this association. Since multiple complications often occur in the same patients, understanding the true impact of any particular complication requires adjustment for all other complications. For example, in unadjusted analyses, we found that vascular complications were associated with higher rates of 1-year mortality and worse 1-year quality of life. However, after adjustment for major bleeding (as well as other complications), neither of these associations remained statistically significant. These findings do not mean that vascular complications are benign but rather indicate that if a vascular complication can be corrected quickly and does not result in a major or life-threatening bleed, then it has little impact on long-term outcomes after TAVR.

Few studies have examined the impact of short-term complications on quality of life after TAVR. A study of 169 German TAVR patients using the Euroqol-5D (a generic health status measure) found quality of life impairment in patients who had strokes, bleeding, or AKI after TAVR (28). A second study of 2,288 patients who underwent TAVR as part of the German Aortic Valve Registry found that stroke and bleeding were both independently associated with worse quality of life (as assessed by the Euroqol-5D) but that renal failure and pacemakers were not (29). To our knowledge, our study is the first to use a diseasespecific health status measure (which is more sensitive to change), to evaluate quality of life alongside mortality, and to examine a broad range of complications (including core-lab determined PVL) using standardized Valve Academic Research Consortium-2 definitions.

\section{Limitations.}

There are a number of potential limitations that should be considered when interpreting our findings. First, since patients had to survive 30 days in order to assess for PVL, early deaths 
were excluded from our primary analysis. However, in a sensitivity analysis that included early deaths (but did not examine PVL), we found similar associations of complications with risk of mortality, although the association between bleeding and mortality was strengthened. Along that same line, quality of life can only be assessed among survivors, so if patients who had serious complications had survived, the quality of life impairments observed with these complications would likely have been even greater. As the associations of the specific complications with mortality and quality of life associations all tracked together, this is likely not a major concern but does highlight the importance of examining these outcomes in combination. Third, the cohort for these analyses included a range of inoperable, high-risk, and intermediate risk patients from a series of nested studies. As such, the frequencies of particular complications should not be generalized to the overall TAVR population, as this question would be best answered in an unselected real-world TAVR cohort. Fourth, although the goal of this study was to examine the association between complications and long-term TAVR outcomes, it is possible that some complications have only a transient effect on quality of life recovery that resolves by 1 year. Finally, the association between complications with outcomes beyond 1 year was not examined. However, inspection of the Kaplan-Meier survival curves showed the association of complications with mortality to be fairly consistent over time, and prior studies have demonstrated that the quality of life benefits of TAVR remain relatively stable beyond 1 year $(30,31)$. As such, it seems unlikely that the associations between complications and 1-year quality of life that we observed would change dramatically beyond 1 year.

\section{CONCLUSIONS}

We found that major stroke and AKI within 30 days after TAVR were associated with a marked increase in the risk of 1-year death and substantial impairment in recovery in quality of life, whereas bleeding and moderate/severe PVL were associated with a more modest increase in long-term mortality and a small but significant quality of life decrement. While continued efforts to reduce the incidence of all TAVR-related complications remain important, these findings suggest that efforts to reduce stroke, AKI, post-procedural bleeding, and PVL should remain key targets for procedural improvement. Conversely, minor stroke, pacemaker implantation, and vascular complications (in the absence of major bleeding) appear to have little long-term impact on patients' recovery after TAVR.

\section{Supplementary Material}

Refer to Web version on PubMed Central for supplementary material.

\section{Funding:}

The PARTNER trial was sponsored by Edwards Lifesciences. This current study was self-funded, and the funding organization for the trial did not play a role in the design and conduct of the study; collection, management, analysis, and interpretation of the data; or preparation, review, or approval of the manuscript. Dr. Arnold is supported by a Career Development Grant Award (K23 HL116799) from the National Heart, Lung, and Blood Institute. 


\section{ABBREVIATIONS AND ACRONYMS}
AKI
acute kidney injury
KCCQ-OS
Kansas City Cardiomyopathy Questionnaire overall summary score
MCS
mental component summary
PARTNER
Placement of AoRTic TraNscathetER Valve
PCS
physical component summary
PVL
paravalvular leak
SF-12
Medical Outcomes Study Short-Form 12 questionnaire
TAVR
Transcatheter aortic valve replacement

\section{REFERENCES}

1. Leon MB, Smith CR, Mack M et al. Transcatheter aortic-valve implantation for aortic stenosis in patients who cannot undergo surgery. N Engl J Med 2010;363:1597-607. [PubMed: 20961243]

2. Reynolds MR, Magnuson EA, Lei Y et al. Health-related quality of life after transcatheter aortic valve replacement in inoperable patients with severe aortic stenosis. Circulation 2011;124:19641972. [PubMed: 21969017]

3. Mack MJ, Leon MB, Smith CR et al. 5-year outcomes of transcatheter aortic valve replacement or surgical aortic valve replacement for high surgical risk patients with aortic stenosis (PARTNER 1): a randomised controlled trial. Lancet 2015;385:2477-84. [PubMed: 25788234]

4. Reardon MJ, Adams DH, Kleiman NS et al. 2-Year Outcomes in Patients Undergoing Surgical or Self-Expanding Transcatheter Aortic Valve Replacement. J Am Coll Cardiol 2015;66:113-21. [PubMed: 26055947]

5. Baron SJ, Arnold SV, Wang K et al. Health Status Benefits of Transcatheter vs Surgical Aortic Valve Replacement in Patients With Severe Aortic Stenosis at Intermediate Surgical Risk: Results From the PARTNER 2 Randomized Clinical Trial. JAMA Cardiol 2017.

6. Leon MB, Smith CR, Mack MJ et al. Transcatheter or Surgical Aortic-Valve Replacement in Intermediate-Risk Patients. N Engl J Med 2016;374:1609-20. [PubMed: 27040324]

7. Arnold SV, Afilalo J, Spertus JA et al. Prediction of Poor Outcome After Transcatheter Aortic Valve Replacement. J Am Coll Cardiol 2016;68:1868-1877. [PubMed: 27765189]

8. Arnold SV, Spertus JA, Vemulapalli S et al. Quality-of-Life Outcomes After Transcatheter Aortic Valve Replacement in an Unselected Population: A Report From the STS/ACC Transcatheter Valve Therapy Registry. JAMA Cardiol 2017;2:409-416. [PubMed: 28146260]

9. Arnold SV, Reynolds MR, Lei Y et al. Predictors of poor outcomes after transcatheter aortic valve replacement: results from the PARTNER (Placement of Aortic Transcatheter Valve) trial. Circulation 2014;129:2682-90. [PubMed: 24958751]

10. Genereux P, Cohen DJ, Williams MR et al. Bleeding complications after surgical aortic valve replacement compared with transcatheter aortic valve replacement: insights from the PARTNER I Trial (Placement of Aortic Transcatheter Valve). J Am Coll Cardiol 2014;63:1100-9. [PubMed: 24291283]

11. Genereux P, Webb JG, Svensson LG et al. Vascular complications after transcatheter aortic valve replacement: insights from the PARTNER (Placement of AoRTic TraNscathetER Valve) trial. J Am Coll Cardiol 2012;60:1043-52. [PubMed: 22883632]

12. Mastoris I, Schoos MM, Dangas GD, Mehran R. Stroke after transcatheter aortic valve replacement: incidence, risk factors, prognosis, and preventive strategies. Clin Cardiol 2014;37:756-64. [PubMed: 25403514] 
13. Pibarot P, Hahn RT, Weissman NJ et al. Association of Paravalvular Regurgitation With 1-Year Outcomes After Transcatheter Aortic Valve Replacement With the SAPIEN 3 Valve. JAMA Cardiol 2017.

14. Regueiro A, Abdul-Jawad Altisent O, Del Trigo M et al. Impact of New-Onset Left Bundle Branch Block and Periprocedural Permanent Pacemaker Implantation on Clinical Outcomes in Patients Undergoing Transcatheter Aortic Valve Replacement: A Systematic Review and Meta-Analysis. Circ Cardiovasc Interv 2016;9:e003635. [PubMed: 27169577]

15. Bagur R, Webb JG, Nietlispach F et al. Acute kidney injury following transcatheter aortic valve implantation: predictive factors, prognostic value, and comparison with surgical aortic valve replacement. Eur Heart J 2010;31:865-74. [PubMed: 20037180]

16. Thourani VH, Kodali S, Makkar RR et al. Transcatheter aortic valve replacement versus surgical valve replacement in intermediate-risk patients: a propensity score analysis. Lancet 2016;387:2218-25. [PubMed: 27053442]

17. Kappetein AP, Head SJ, Genereux P et al. Updated standardized endpoint definitions for transcatheter aortic valve implantation: the Valve Academic Research Consortium-2 consensus document. J Am Coll Cardiol 2012;60:1438-54. [PubMed: 23036636]

18. Arnold SV, Spertus JA, Lei Y et al. Use of the kansas city cardiomyopathy questionnaire for monitoring health status in patients with aortic stenosis. Circ Heart Fail 2013;6:61-7. [PubMed: 23230306]

19. Spertus J, Peterson E, Conard MW et al. Monitoring clinical changes in patients with heart failure: a comparison of methods. Am Heart J 2005;150:707-15. [PubMed: 16209970]

20. Spertus J, Peterson E, Conard MW et al. Monitoring clinical changes in patients with heart failure: a comparison of methods. Am Heart J 2005;150:707-15. [PubMed: 16209970]

21. Ware J, Jr., Kosinski M, Keller SD. A 12-Item Short-Form Health Survey: construction of scales and preliminary tests of reliability and validity. Med Care 1996;34:220-33. [PubMed: 8628042]

22. Arnold SV, Lei Y, Reynolds MR et al. Costs of periprocedural complications in patients treated with transcatheter aortic valve replacement: results from the Placement of Aortic Transcatheter Valve trial. Circ Cardiovasc Interv 2014;7:829-36. [PubMed: 25336467]

23. Popma JJ, Adams DH, Reardon MJ et al. Transcatheter aortic valve replacement using a selfexpanding bioprosthesis in patients with severe aortic stenosis at extreme risk for surgery. $\mathrm{J}$ Am Coll Cardiol 2014;63:1972-81. [PubMed: 24657695]

24. Grover FL, Vemulapalli S, Carroll JD et al. 2016 Annual Report of The Society of Thoracic Surgeons/American College of Cardiology Transcatheter Valve Therapy Registry. J Am Coll Cardiol 2017;69:1215-1230. [PubMed: 27956264]

25. Abdel-Wahab M, Mehilli J, Frerker C et al. Comparison of balloon-expandable vs self-expandable valves in patients undergoing transcatheter aortic valve replacement: the CHOICE randomized clinical trial. JAMA 2014;311:1503-14. [PubMed: 24682026]

26. Chakravarty T, Sondergaard L, Friedman J et al. Subclinical leaflet thrombosis in surgical and transcatheter bioprosthetic aortic valves: an observational study. Lancet 2017;389:2383-2392. [PubMed: 28330690]

27. Makkar RR, Fontana G, Jilaihawi H et al. Possible Subclinical Leaflet Thrombosis in Bioprosthetic Aortic Valves. N Engl J Med 2015;373:2015-24. [PubMed: 26436963]

28. Kaier K, Gutmann A, Baumbach $\mathrm{H}$ et al. Quality of life among elderly patients undergoing transcatheter or surgical aortic valve replacement- a model-based longitudinal data analysis. Health Qual Life Outcomes 2016;14:109. [PubMed: 27456092]

29. Lange R, Beckmann A, Neumann T et al. Quality of Life After Transcatheter Aortic Valve Replacement: Prospective Data From GARY (German Aortic Valve Registry). JACC Cardiovasc Interv 2016;9:2541-2554. [PubMed: 28007203]

30. Baron SJ, Arnold SV, Wang K et al. Health Status Benefits of Transcatheter vs Surgical Aortic Valve Replacement in Patients With Severe Aortic Stenosis at Intermediate Surgical Risk: Results From the PARTNER 2 Randomized Clinical Trial. JAMA Cardiol 2017;2:837-845. [PubMed: 28658491] 
31. Baron SJ, Arnold SV, Reynolds MR et al. Durability of quality of life benefits of transcatheter aortic valve replacement: Long-term results from the CoreValve US extreme risk trial. Am Heart $\mathbf{J}$ 2017;194:39-48. [PubMed: 29223434] 


\section{PERSPECTIVES}

\section{WHAT IS KNOWN?}

Due to the age and comorbidities of patients who undergo TAVR, complications are not infrequent, and many of these complications have, in isolation, been shown to be associated with increased mortality.

\section{WHAT IS NEW?}

We found that major stroke and stage 3 kidney injury, while occurring infrequently, have a marked impact on survival and quality of life (even after taking into account patient factors and concomitant complications); whereas bleeding and paravalvular regurgitation, which occur more commonly, have a more modest negative impact on outcomes.

\section{WHAT IS NEXT?}

Further studies are needed to understand both the frequency of complications and their impact on outcomes in patients in routine clinical practice and to monitor how these change over time, with innovations in devices and periprocedural care. 
Table 1.

\section{Baseline Patient Characteristics}

\begin{tabular}{lc}
\hline & $\mathbf{n}=\mathbf{3 7 6 3}$ \\
\hline Age $(\mathrm{y})$ & $82.0 \pm 7.8$ \\
Female & $1573(41.8 \%)$ \\
Coronary artery disease & $2663(70.8 \%)$ \\
Prior coronary bypass surgery & $1176(31.0 \%)$ \\
Prior coronary stenting & $1168(31.3 \%)$ \\
Prior stroke or TIA & $697(18.5 \%)$ \\
Peripheral vascular disease & $1151(30.6 \%)$ \\
Atrial fibrillation/flutter & $1448(38.5 \%)$ \\
Diabetes mellitus & $1322(35.1 \%)$ \\
Oxygen-dependent lung disease & $289(7.7 \%)$ \\
Estimated GFR (mL/min/1.73 $\left.\mathrm{m}^{2}\right)$ & $61.9 \pm 243.2$ \\
Renal insufficiency $(\mathrm{Cr} 2)$ & $335(8.9 \%)$ \\
Ejection fraction $(\%)$ & $55.2 \pm 13.5$ \\
Body mass index $\left(\mathrm{kg} / \mathrm{m}^{2}\right)$ & $28.4 \pm 6.4$ \\
Dementia & $117(3.1 \%)$ \\
Hematocrit $(\%)$ & $36.6 \pm 7.6$ \\
Mean aortic valve gradient $(\mathrm{mmHg})$ & $44.2 \pm 13.9$ \\
Aortic valve area $\left(\mathrm{cm}{ }^{2}\right)$ & $0.71 \pm 0.22$ \\
STS Mortality Risk Score $(\%)$ & $7.4 \pm 4.0$ \\
Transfemoral approach & $3009(80.0 \%)$ \\
KCCQ-OS score & $49.6 \pm 22.6$ \\
SF-12 Physical Summary score & $35.1 \pm 9.2$ \\
SF-12 Mental Summary score & $48.5 \pm 11.7$ \\
\hline
\end{tabular}

TIA, transient ischemic attack; GFR, glomerular filtration rate; Cr, creatinine; STS, Society of Thoracic Surgery; KCCQ-OS, Kansas City Cardiomyopathy Questionnaire-Overall Summary; SF-12, 12-item Medical Outcomes Short Form 
Table 2.

\begin{tabular}{|c|c|}
\hline & $n=3763$ \\
\hline Major stroke & $58(1.5 \%)$ \\
\hline Minor stroke & $54(1.4 \%)$ \\
\hline Life-threatening bleed & $259(6.9 \%)$ \\
\hline Major bleed & $631(16.8 \%)$ \\
\hline Major vascular event & $243(6.5 \%)$ \\
\hline New pacemaker & $326(8.7 \%)$ \\
\hline Acute kidney injury stage 3 & $39(1.0 \%)$ \\
\hline Moderate/severe paravalvular leak & $269(8.1 \%)$ \\
\hline Mild paravalvular leak & $1155(34.7 \%)$ \\
\hline
\end{tabular}

D্

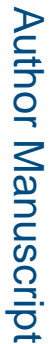

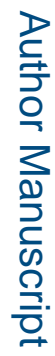

JACC Cardiovasc Interv. Author manuscript; available in PMC 2020 February 25. 


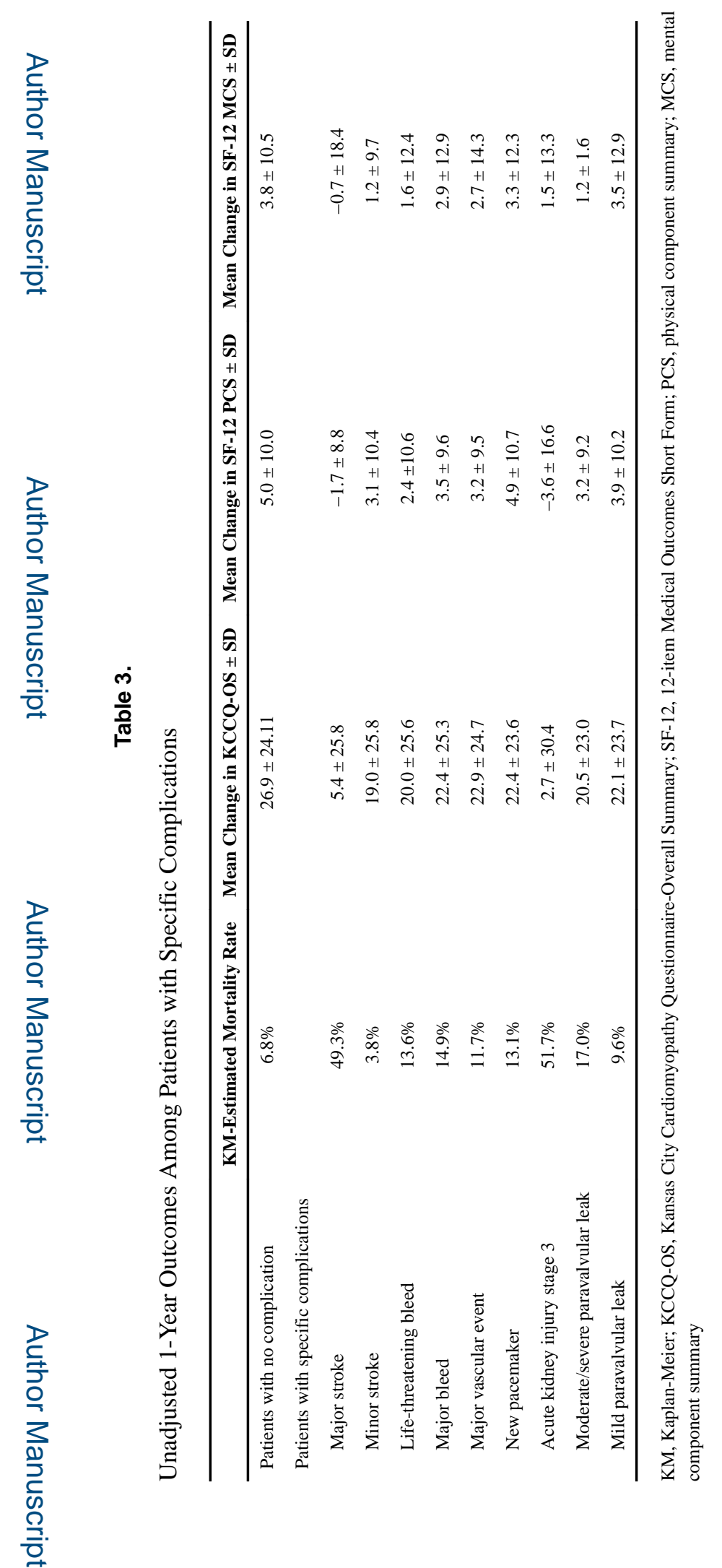

JACC Cardiovasc Interv. Author manuscript; available in PMC 2020 February 25. 


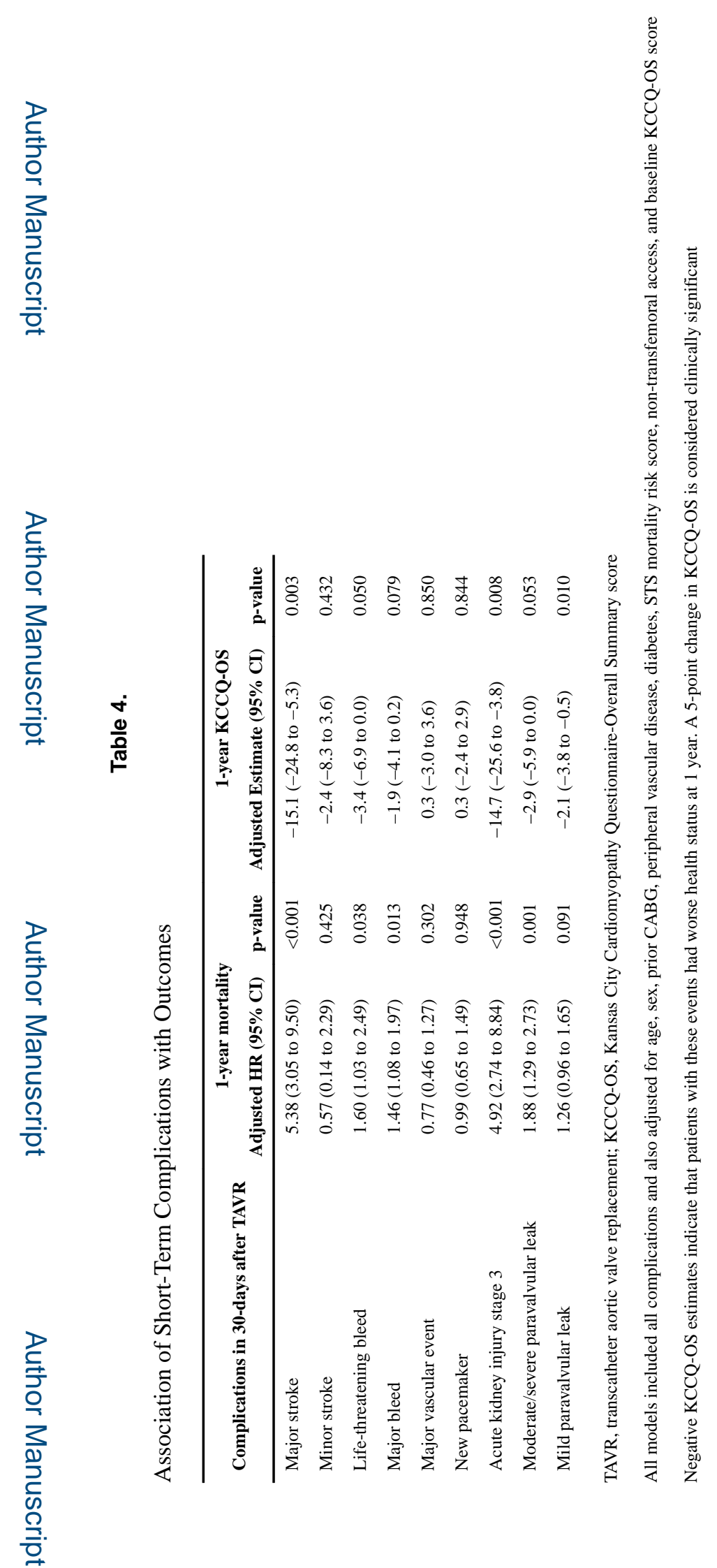

JACC Cardiovasc Interv. Author manuscript; available in PMC 2020 February 25. 